\title{
Relations des Virus d'animaux et des Rickettsies avec leurs Tiques vectrices
}

\author{
par P.-C. MOREL \\ Institut d'Elevage et de Médecine Vétérinaire des Pays Tropicaux \\ 10, rue Pierre-Curie - F 94 -Maisons-Alfort
}

\begin{abstract}
Résumé
Les tiques spécifiquement réceptives à une rickettsie le sont à tous les stades. Les pourcentages d'infections selon les stades sont fonction de la qualité de sang ingéré et de la concentration du sang en rickettsies.

Les tiques présentent une infection générale, entraînant rarement leur mort, avec les genres Rickettsia et Coxiella; une infection uniquement intestinale avec les genres Cowdria et Rakeia; le genre Wolbachia, déterminant une infection générale non pathogène, ne comprend que des espèces symbiotes d'insectes et d'acariens. L'association rickettsies-tiques semble donc primitive et le parasitisme des vertébrés une conséquence de l'hématophagie des arthropodes.

Les voies ordinaires d'élimination des rickettsies sont les sécrétions salivaires et les excreta (ainsi que le liquide coxal, les Argasidae). L'infection dure toute la vie de la tique et se transmet de stade à stade à travers les mues.

L'infection par la voie transovarienne de la descendance d'une femelle infectée s'observe couramment chez Coxiella et Rickettsia, ainsi que chez Anaplasma. Cette possibilité est liée à l'infection générale de la tique.

Le jeûne, l'hibernation, les métamorphoses entraînent en général une baisse de virulence des souches rickettsiennes; cette virulence est restaurée par repas de sang ou séjour à $37-38^{\circ} \mathrm{C}$.

Du point de vue de l'épidémiologie des rickettsioses, les tiques doivent surtout être considérées comme des agents de transport et de conservation sur plusieurs générations. La transmission directe des rickettsies par piqûre ne doit vraisemblablement pas jouer un
\end{abstract}


rôle plus important que la contamination de la lésion cutanée par les matières fécales de l'acarien ou leur dissémination sous forme de poussières dans le pelage des animaux et dans l'atmosphère.

Les tiques spécifiquement réceptives à un ultra-virus le sont à tous les stades. Les pourcentages d'infections selon les stades sont en fonction de la quantité de sang ingéré et de la concentration du sang en virus (notion de seuil d'infection).

Les tiques véritablement réceptives à un virus présentent une infection générale; le passage à travers la barrière intestinale correspond à un caractère génétique d'adaptation entre le virus et une espèce de tique. Cette infection générale n'entraîne pas la mort de l'acarien hôte.

L'infection transovarienne de la descendance ne s'observe que dans un nombre limité de cas et n'a certainement qu'une importance secondaire dans l'épidémiologie des arboviroses à tiques.

Il n'y a pas de modification de virulence pour une souche de virus au cours du cycle chez la tique.

Du point de vue de l'épidémiologie des arboviroses, les tiques sont considérées comme des vecteurs directs par piqûre de nymphe ou d'adulte infectés au stade précédent.

\section{Summary}

The ticks specifically susceptible to a rickettsia are so at every stage. According the stages, the infection rates are related with the quantity of ingested blood and the blood concentration of rickettsiae.

Ticks present a general infection leading rarely to death with the genera Rickettsia and Coxiella; a merely intestinal infection occurs with the genera Rakeia and Cowdria; the genus Wolbachia, determining a general non pathogenic infection, includes only species symbiotic with insects and acari. The association between rickettsiae and ticks therefore seems to be primitive, and the parasitism of the vertebrates a consequence of the hematophagy of the arthropods.

The ordinary elimination ways of rickettsiae are the salivary secretions and excreta (so as the coxal fluid of Argasidae). Infection lasts during the whole life of the tick and is transmitted from stage to stage through the molting periods.

The transovarial way of infection of the progeny from an infected female is usually observed by Coxiella and Rickettsia, so as by Anaplasma. That possibility is related to the general infection of the tick.

Fasting, hibernation and metamorphosis generally lead to a diminution of virulence in the rickettsial strain. This virulence is restored by a blood meal, or by remaining at $37-38^{\circ} \mathrm{C}$. 
From the epidemiological point of view of the rickettsiosis, ticks are to be considered as transportation and conservation agents over various generations. Direct transmission by biting does not likely take a part more important than the contamination of the skin lesions by the excreta of the ticks or their spreading in dusty form in the fur or in the atmosphere.

The ticks specifically susceptible to a virus are so at every stage. According the stages, the infection rates are related with the quantity of ingested blood and the blood concentration of viruses (notion of threshold infection).

The really susceptible ticks to a virus present a general infection; the passage through the gut barrier responds to a genetical character of adaptation between a virus and a species of tick. That general infection does not lead to the death of the host tick.

The transovarial infection of the progeny is only observed in a limited number of cases and seems of secondary importance in the epidemiology of the tick transmitted arboviroses.

There is no modification of virulence for a virus strain during the cycle in the tick.

From the epidemiological point of view of the arboviroses, ticks are to be considered as direct vectors, by biting of nymphs or adults infected in the preceding stage.

Dans la plus grande part des publications traitant des rapports entre ultra-virus, rickettsies et tiques, les auteurs s'efforcent de reconnaître les vecteurs naturels, afin de dégager de l'écologie et du comportement de ces derniers les données perméttant de comprendre l'épidémiologie des affections dues aux agents pathogènes transmis, et prévoir un système de prophylaxie basé sur la lutte directe contre le vecteur. Dans cette optique, les études sont menées conjointement par l'entomologiste et le microbiologiste.

Or, si la situation et le comportement du vecteur doivent être connus, ainsi que les conditions de réceptivité ou d'immunité d'un vertébré sensible à un agent pathogène donné, la connaissance des réactions physiologiques et pathologiques parallèles, qui ont lieu chez l'arthropode, est également indispensable pour concevoir dans sa totalité les modalités de circulation du virus au niveau de ses divers relais. C'est ainsi que l'étude en laboratoire des aptitudes d'un arthropode donné à permettre la survie et la multipliplication d'un virus donné, loin de représenter une recherche théorique seulement bonnè à orienter le travail sur le terrain, fournit justement les renseignements rendant possible l'interprétation des faits plus ou moins coordonnés recueillis au cours des enquêtes.

C'est ainsi que sur le nombre des références concernant la transmission des ultravirus et des rickettsies par les tiques, il s'agit le plus souvent de constatations d'infection de telle ou telle espèce d'acarien recueillie dans la nature. Au contraire, les textes sur les réactions propres d'une tique à un agent donné sont beaucoup moins nombreux. On peut dire en pratique que commencent à être mieux connues les relations entre Rickettsia rickettsi et Dermacentor andersoni ou quelques Argasidae, entre Coxiella burneti et divers Ixodidea d'Australie ou des Etats-Unis entre le virus de l'encéphalite à tiques 
européennes et Ixodes ricinus, entre le virus de l'encéphalite de la taïga et Ixodes persulcatus, entre le virus de Kyasanur et quelques Haemaphysalis. Dans le cas de beaucoup d'autres agents pathogènes, le plus souvent on ne peut que se contenter de généraliser en se basant sur les schémas de rapports virus-vecteur précités.

A titre d'exemple, quelques faits de cet ordre sont résumés dans un tableau portant sur les ultra-virus et rickettsies associés aux tiques en Europe occidentale et dans le bassin méditerranéen ; il y apparaît que les connaissances y sont réduites et fragmentaires. Pour déborder ce cadre géographique et avoir cité l'essentiel de ces relations entre virus et acariens, il faut ajouter le virus de l'encéphalite équine du Proche-Orient (isolé de Hyalomma excavatum), le virus West Nile (dont une partie du cycle peut mettre en jeu des Argasidae), des virus comme Chenuda et Qaranfil (infectant naturellement des Argas hermanni et $A$. arboreus parasites d'oiseaux sauvages en Egypte), le virus de Nairobi (transmis au mouton par Rhipicephalus appendiculatus), Rickettsia akari (et les Dermanyssidae), Rickettsia tsutsugamushi (et les Trombiculidae), Rickettsia australis du Queensland et $R$. sibirica de Sibérie (et plusieurs tiques de ces contrées), Cowdria ruminantium et des Amblyomma d'Afrique éthiopienne.

Une difficulté dans l'étude des Rickettsia par les méthodes sérologiques vient de l'inadaptation de la nomenclature en usage à la systématique véritable du genre ; les noms créés pour désigner un agent pathogène associé à un vecteur particulier $(R$. prowa$z e k i$ du pou, $R$. mooseri des puces) ou à une région géographique donnée ( $R$. conori d'Afrique et Eurasie occidentale, $R$. sibirica de Sibérie, $R$. rickettsi des deux Amériques, $R$. australis d'Australie), ne représentent certainement pas autre chose que des souches plus ou moins apparentées d'une même espèce, et différenciées par mutation à partir d'un fond génétique commun, en relation avec la transmission par un hôte donné ou avec un isolement géographique; les résultats inconstants de la sérologie indiquent un grand nombre de souches de virulences diverses circulant plus ou moins librement par l'intermédiaire d'arthropodes, insectes ou acariens, très variés.

Il faut signaler enfin l'existence de Rickettsiales purement symbiotes de tiques et non pathogènes pour les vertébrés : Wolbachia dermacentrophila de Dermacentor andersoni et $W$. persica d'Argas arboreus; ces éléments sont surtout abondants à l'intérieur des cellules des tubes de Malpighi, des glandes salivaires, des ovocytes et du réseau trachéen; des Wolbachia non dénommées ont été observées chez d'autres tiques. Des Wo!bachia ont été également décrites chez les insectes.

La question des rapports des tiques avec l'épidémiologie des rickettsioses et des viroses humaines a été résumée dans deux textes de $\mathrm{H}$. Hoogstraal : Ticks in relation to human diseases caused by viruses, Ann. Rev. Entom., 1966, 11: 261-308 ; Ticks in relation to human diseases caused by Rickettsia, species, Ann. Rev. Entom., 1967, 12 : 377-420. Il est indispensable de s'y reporter.

Les relations des ultra-virus et des rickettsies avec les tiques, dont l'étude doit constituer la partie principale du présent rapport, ont déjà fait l'objet d'une revue critique par J. Reháček: Development of animal viruses and Rickettsiae in ticks and mites, Ann. Rev. Entom., 1965, 10:1-24. C'est à ce texte que sont empruntées la plupart des données. 


\begin{tabular}{|c|c|c|c|c|c|c|c|c|c|c|c|c|c|c|c|c|c|}
\hline 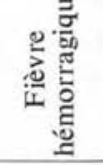 & & 莺 & $\mathbf{Z}$ & $\begin{array}{l}\vec{g} \\
z\end{array}$ & & & & 苋 & $\mathbf{Z}$ & & & & & & & & \\
\hline 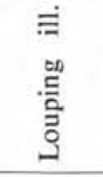 & & & & & & 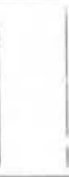 & & & & & & & & & $\begin{array}{c}E \\
\stackrel{E}{*} \\
\vec{a} \\
z\end{array}$ & & \\
\hline 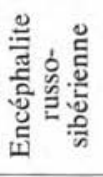 & $\begin{array}{l}\underset{\omega}{*} \\
\vec{w} \\
z\end{array}$ & & $\begin{array}{l}\overrightarrow{0} \\
\mathbf{z}\end{array}$ & $\begin{array}{l}\overrightarrow{3} \\
\text { z } \\
\text { z }\end{array}$ & & & & & & & & & & & 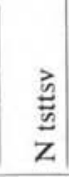 & & \\
\hline 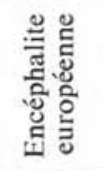 & & & & & & & & & & & & & & & 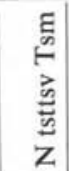 & $\underline{\underline{z}}$ & \\
\hline 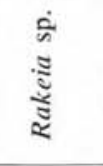 & & & $\mathbf{Z}$ & & & & $\mathbf{z}$ & & & & & & z & & z & $z$ & \\
\hline 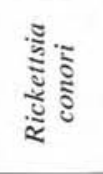 & & Z & $\mathrm{z}$ & z & & & & & & & z & 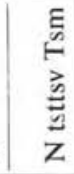 & Z & 'Z & & & \\
\hline 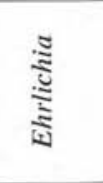 & & & & & & & $\begin{array}{c}E \\
f \\
f^{n} \\
\delta z\end{array}$ & & & 后 & & 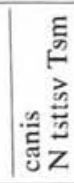 & & & & & \\
\hline ปั: & $\underline{\underline{2}}$ & z & $\begin{array}{l}\vec{y} \\
\frac{y}{y} \\
z \\
z\end{array}$ & $\vec{\otimes}$ & z & $\mathbf{z}$ & $\begin{array}{l}\frac{\overrightarrow{3}}{3} \\
\frac{5}{2} \\
z\end{array}$ & $\mathbf{Z}$ & $\frac{\vec{w}}{z}$ & $\begin{array}{l}\overrightarrow{3} \\
\frac{3}{2} \\
z\end{array}$ & z & $\begin{array}{l}E \\
\stackrel{5}{*} \\
\vec{a} \\
z\end{array}$ & $\begin{array}{l}\overrightarrow{3} \\
\frac{n}{2} \\
\mathrm{z}\end{array}$ & $\mathrm{z}$ & $\mathbf{Z}$ & & $\begin{array}{l}\vec{\Phi} \\
z\end{array}$ \\
\hline & 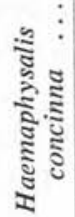 & 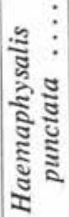 & 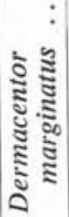 & 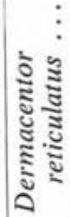 & 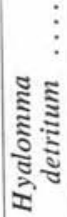 & 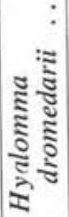 & 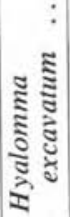 & 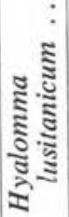 & 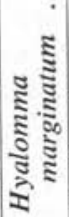 & 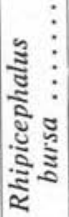 & 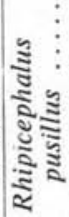 & 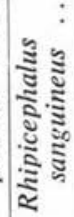 & 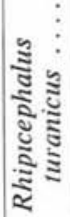 & 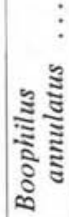 & 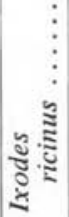 & 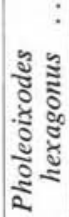 & 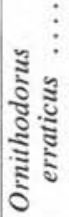 \\
\hline
\end{tabular}


La voie d'entrée des ultra-virus et rickettsies dans l'organisme des tiques est l'hématophagie ; dans la nature il s'agit de sang d'un animal en virémie ou rickettsiémie ; au laboratoire, il peut s'agir d'un liquide artificiel contenant un agent pathogène au titre connu absorbé suivant des dispositifs variés (membranes, tubes capillaires). Le passage par le pharynx et l'œsophage est très rapide et ces organes ne peuvent pas représenter un accès vers l'intérieur de l'organisme de l'arthropode.

On ne connaît pas le mécanisme de la résistance des tiques à un virus ou une rickettsie; il ne semble pas qu'il s'agisse d'anticorps arthropodiens; par ailleurs, les anticorps du scng de l'hôte jouent un rôle important dans le devenir de l'infection de la tique acquise au cours d'un précédent stade ou en train de l'être au cours du dernier repas.

\section{Ultra-virus et Tiques}

\section{Infection intestinale.}

Le premier contact prolongé du virus avec les cellules de l'hôte a lieu au niveau de l'épithélium digestif. Ce contact se produit au cours de diverses transformations de la masse sanguine ingérée. Le sang subit d'abord une concentration, par élimination d'eau et de sels minéraux ; la déshydratation est assurée par l'épithélium et les éléments éliminés passent dans l'hémolymphe à travers la membrana propria et l'assise musculaire : ils sont ensuite excrétés, chez les Ixodida par transsudation au niveau de cellules cuticulaires, chez les Argasida par sécrétion rapide des glandes coxales. Les hématies subissent une hémolyse avant d'être digérées dans la lumière du tube digestif ou à l'intérieur des cellules épithéliales amoeboïdes fixées ou libres ; les cristaux d'hémoglobine s'accumulent entre les hématies.

C'est la réaction propre de l'épithélium intestinal qui détermine la possibilité d'infection de l'arthropode. Les facteurs conditionnant la réceptivité de l'arthropode sont d'ordre génétique (un virus transmis par plusieurs vecteurs ne l'est pas par chacun dans les mêmes conditions ; les différences se situent au niveau spécifique ou générique) ; chez les espèces réceptives interviennent de plus les facteurs trophiques, qui régissent le degré d'infection (importance de la dose de virus ingérée corrélative à la quantité de sang absorbé). Il existe un seuil d'infection correspondant à la quantité minimale de virus nécessaire à infecter l'épithélium intestinal.

Ces considérations se situent sur un plan théorique ; les essais en laboratoire mettent en jeu des acariens infectés expérimentalement et ne préjugent pas du rôle réel de l'éspèce dans l'épidémiologie ; on met en évidence une réceptivité potentielle. L'épidémiologiste utilisera ces données absolues pour les intégrer dans le système des relations entre l'arthropode, les réservoirs de virus et les vertébrés sensibles à ce virus.

Le rôle de l'épithélium digestif dans l'infection de l'arthropode est fondamental; il existe en effet une différence entre la réceptivité naturelle à l'infection par un repas de sang infectieux, et la réceptivité à la suite d'une injection parentérale d'un agent patho- 
gène. On infecte de cette façon des tiques avec des virus qui n'ont jamais été infectants far voie digestive.

Après le repas infectieux, la présence de virus dans le tube digestif ne signifie pas que la tique a été infectée; le virus peut demeurer dans la lumière intestinale et être longtemps excrété ; il peut y avoir survie sans multiplication dans l'épithélium. Ainsi le virus de l'encéphalomyélite équine de l'Est n'effectue qu'un passage intestinal chez Ixodes ricinus ou Dermacentor marginatus au contraire du virus de l'encéphalite à tiques (Reháček, 1959, 688). Finalement, les virus sont inactivés et disparaissent.

\section{Infection fécale.}

L'infection fécale est une conséquence de la simple survie du virus dans l'intestin ou de l'infection intestinale véritable. L'hémoglobine cristallisée protège le virus dans les excréments. L'élimination de virus par cette voie se produit lors de tous les repas de sang virémique.

La survie d'un virus est longue dans les matières fécales, beaucoup plus que sa résistance normale dans d'autres produits organiques.

Pendant les périodes de repos, les virus ne sont plus excrétés. Au cours des repas suivants, l'excrétion reprendra, et le taux du virus dépend du degré de réinfection de la tique au cours du repas (voir la transmission transstadiale). Si l'hôte est immun et si son sang contient des anticorps, le virus n'est plus excrété.

\section{Infection générale.}

Elle provient d'une extension de l'infection intestinale qui en est donc la condition fondamentale. Dans le transport de l'agent, le rôle des cellules fixes comme les oenocytes et néphrocytes du réseau trachéen, à aptitudes phagocytaires, n'est pas confirmé, non plus que celui des hémocytes mobiles de l'hémolymphe.

Dans le cas du virus de l'encéphalite de la taïga, tous les organes d'Ixodes persulcatus en sont atteints dès le lendemain du repas infectieux; les titres de virus sont élevés dans l'épithélium digestif, les glandes salivaires et les ovaires, où se produit une concentration ; les titres sont faibles dans les tubes de Malpighi, les ganglions cérébroïdes, l'organe de Gené et l'hémolymphe. Chez les Argasidae, le virus peut même passer dans le liquide coxal par les hémocytes, qui pourraient ainsi servir à diffuser le virus dans le corps de la tique ; c'est le cas du virus West Nile chez Ornithodorus moubata infecté par voie parentérale.

\section{Persistance du virus chez les tiques.}

Au cours de la survie du virus se produisent plusieurs cycles de multiplication, puis le virus disparaît. Ce temps de survie peut être très long, de 9 mois pour le virus de l'encéphalite à tiques chez la femelle d'Ixodes ricinus, à $4^{\circ} \mathrm{C}$ (Benda, 1958, 314), de 14 mois pour le virus du louping ill chez les femelles d'I. ricinus (Mac Leod, 1962, 411), de 8 mois chez les larves, de 13 mois chez les nymphes, de 29 mois chez les femelles de 
Rhipicephalus appendiculatus pour le virus de Nairobi du mouton (Lewis, 1946, 55). Dans la nature, les temps de survie peuvent être très longs dans des circonstances favorables. Par ailleurs, les temps de vie réelle des tiques doivent être inférieurs à ceux où ces tiques perdraient leur infection virale.

Chez des Gamasidae expérimentalement infectés des virus de Saint-Louis, de l'encéphalomyélite équine, de la chorioméningite lymphocytaire, la survie du virus est très courte.

\section{Pathologie des vecteurs par les Virus.}

Il ne semble pas que les ultra-virus déterminent des troubles pathologiques chez les tiques. Tout au moins, les virus de l'encéphalite européenne et de l'encéphalite de la taïga n’ont pas affecté les divers stades d'Ixodes ricinus (Reháček, 1965, 1-24).

\section{Infection transstadiale.}

Pendant les métamorphoses, ce sont surtout les tissus ectodermiques qui sont touchés, beaucoup moins les organes internes tels que l'intestin, les tubes de Malpighi ou les ovaires. Cette absence d'histolyse du tube digestif assure l'infection transstadiale.

Du fait de leur type de sécrétion holocrine, les glandes salivaires dégénèrent et disparaissent; le virus doit envahir les glandes néoformées au stade suivant.

L'infection transstadiale semble la règle chez les tiques porteuses de virus. Les variations du titre viral dans l'organisme de la tique ont lieu suivant certaines modalités selon les espèces.

Chez les larves d'Ixodes ricinus, il y a augmentation de la quantité de virus, par multiplication à l'intérieur des cellules, dans les 2-4 semaines après le repas infectant de virus de l'encéphalite européenne, puis cette valeur décroît et reste stationnaire jusqu'à la métamorphose ; à l'éclosion de la nymphe, le titre a encore baissé ; le titre ne remonte qu'au repas nymphal, soit du fait d'un changement de métabolisme de la tique, soit par réinfection par le sang infectieux de l'hôte; finalement, le pourcentage des adultes infectés issus de nymphes infectées atteint $60-90$ p. 100 ; l'infection des larves parvient jusqu'aux adultes, même si le repas nymphal a eu lieu sur un hôte neuf. La multiplication du virus est plus importante chez les tiques nouvellement infectées que chez celles infectées à un stade précédent (Benda, 1958, 314).

Les anticorps spécifiques ingérés avec le sang d'un hôte immun interviennent dans l'évolution de l'infection chez la tique. Cette action se produit au niveau du tube digestif et des autres organes de la tique et se poursuit au-delà des métamorphoses. Ainsi des nymphes d'l. ricinus infectées du virus d'encéphalite européenne perdent leur infection si leur repas a lieu sur un hôte immun ; d'autre part, les tiques ne peuvent plus être réinfectées ultérieurement (Benda, 1958, 314).

Avec d'autres virus, l'infection reste stationnaire ou décroît après le repas, et la multiplication n'intervient qu'après la métamorphose, soit sous l'influence des hormones de pupaison, soit du fait de la jeunesse des nouveaux tissus. C'est le cas pour le virus du Colorado chez Dermacentor andersoni (Rozeboom et Burgdorfer, 1959, 138) ou 
celui du virus de Kyasanur chez Haemaphysalis spinigera (Varma et Smith, 1960, 397) ; dans ce dernier cas, l'infection des larves n'est parfois décelable que chez les nymphes nouvellement écloses.

\section{Infection transovarienne.}

Il existe dans les tissus de l'ovaire une défense vis-à-vis des ultra-virus qui fait qu'ils sont très rarement envahis. Le phénomène ne s'observe que dans 6 p. 100 des larves d'Ixodes ricinus issues de femelles infectées du virus de l'encéphalite européenne dans les conditions expérimentales; le pourcentage est nul si le repas de la femelle a eu lieu sur hôte immun (Benda, 1958, 314). La voie expérimentale a son influence, car il y a eu 3 p. 100 d'infection transovarienne à partir de femelle infectées par repas, mais 20 p. 100 à partir de femelles infectées par voie parentérale (Reháček, 1695, 220).

$\mathrm{Au}$ total on peut estimer que l'infection transovarienne se réalise rarement dans la nature et n'a pas de signification épidémiologique.

\section{Transmission des Virus.}

La transmission du virus par la tique à l'hôte sensible se fait au cours du repas. Dans le cas du long repas des Ixodida, une virémie s'installe plus ou moins rapidement suivant la réceptivité, avant la fin du repas de la tique ; celle-ci peut donc se réinfecter par le sang virulent. La quantité de virus produite est fonction de la taille de la tique. Les piqûres de 4-5 femelles d'Ixodes ricinus suffisent à provoquer une virémie chez la chèvre, suivie d'élimination dans le lait (Benda, 1958, 1-5). Quelques larves (2-5 d'I. persulcatus, 3-7 d'I. ricinus) suffisent à provoquer une encéphalite chez la souris en 2-3 jours (Petriščeva et Levkovič, 1949, 42).

Les possibilités de transmission par repas interrompu, commencé sur un hôte virulent et terminé sur un hôte neuf, existent, mais représentent un phénomène peu important pour les femelles dans la nature; chez les mâles, qui changent souvent de place et d'hôtes, il en va peut-être autrement.

Le pouvoir infectant de la tique est lié à la quantité de virus émise, c'est-à-dire à son stade.

Chez les Argasidae, il existe également la possibilité d'infection par le liquide coxal ; le fait a été observé avec Ornithodorus moubata infecté de virus West Nile (Hurlbut, 1956, 76 ; Vermeil, Lavillaureix et Reeb, 1960, 273 ; Whitman et Aitken, 1960, 192).

\section{Changements de propriété des Virus.}

Il n'y a pas de référence indiquant à l'heure actuelle un changement de virulence chez un virus au cours du passage chez une tique.

Ainsi, des souches de virulences diverses existent pour le virus de l'encéphalite de la taïga, mais elles conservent leurs caractéristiques au cours des passages par les tiques (Libikova et coll., 1964, 77). Il en est de même pour le virus de l'encéphalite européenne chez I. ricinus; on a observé très peu de différences antigéniques entre des 
souches conservées à $4^{\circ} \mathrm{C}$ ou à $18-20^{\circ} \mathrm{C}$ pendant 9 mois (Benda, 1958, 314). Le même virus a subsisté 102 jours chez des larves d' $I$. ricinus en hibernation à $4^{\circ} \mathrm{C}$ et 10 mois chez des nymphes à jeun, sans varier de virulence pour la souris (Rehaček, 1960, 106). Mêmes résultats pour le virus West Nile, après 188 jours chez des nymphes I et II d'Ornithodorus moubata (Whitman et Aitken, 1960, 192).

\section{Rickettsies et Tiques}

Il y a de nombreux points communs dans leurs relations avec les tiques entre les rickettsies et les virus, si bien que les commentaires sur les virus sont en partie valables en ce qui concerne les rickettsies.

\section{Infection intestinale.}

Elle se réalise de la même façon que chez les virus. Dans certains cas, elle est seule en jeu, les rickettsies n'envahissant pas l'organisme, malgré leur présence dans l'épithélium digestif. Ainsi Coxiella burneti chez les Argasidae et Cowdria ruminantium chez les Amblyomma.

\section{Infection fécale.}

Coxiella burneti présente une résistance très longue dans les matières fécales de l'acarien ; on l'a observée jusqu'à 586 jours chez Dermacentor andersoni (Philip, 1948, 457), jusqu'à 744 jours chez Ornithodorus tholozani (Daiter et Amosenkova, 1961, 166). En revanche, la persistance est faible chez Rickettsia rickettsi (Philip, 1959, 595).

De toute façon, cette possibilité pour les rickettsies de survivre un temps plus ou moins long sous forme de poussières, provenant de la dessication des matières fécales de la tique, dans le pelage des animaux ou dans les vêtements ou sur la peau de l'homme, est un facteur favorisant très important dans l'épidémiologie des rickettsioses.

\section{Infection générale.}

Une méthode actuelle extrêmement précise dans l'étude de la localisation des rickettsiales dans l'organisme des arthropodes est l'utilisation d'anticorps fluorescents, sur coupes au cryostat, sans fixation physico-chimique. La microscopie électronique est un autre moyen d'étude.

Après l'infection primaire du tube digestif, les rickettsies sont isolées des tubes de Malpighi, ovaires, ganglions cérébroïdes, glandes salivaires, testicules, aussi bien chez des tiques récoliées dans la nature que dans des élevages de $2^{\circ}$ génération au laboratoire (Coxiella burneti, Rickettsia conori, R. sibirica).

Chez les Argasidae infectés par la voie parentérale, les rickettsies se retrouvent dans le contenu intestinal et le liquide coxal (C. burneti chez Ornithodorus moubata; Weyer. 
1953, 344) ou dans l'hémolymphe (Rickettsia australis chez O. moubata; Weyer, 1959, 609).

C. burneti injectée à Hyalomma marginatum par voie paarentérale, se retrouve le lendemain dans les cellules du tube digestif, 3 jours après dans les ovaires et les glandes salivaires ; la multiplication se poursuit pendant 25 jours dans les organes et les hémocytes ; ces derniers semblent bien responsables de la diffusion de $C$. burneti chez la tique (Tarasevič, 1957, 818).

Chez divers Ixodida, Kordova et Rehaček $(1964,465)$ observent Rickettsia prowazeki dans les hémocytes dès le lendemain de l'injection parentérale ; jusqu'au $9^{\circ}$ jour apparaissent dans le cytoplasme des hémocytes et de diverses cellules des vacualisations, granulations et formes granuleuses; les formes typiques sont présentes dans les cellules de l'hypoderme et du réseau trachéen à partir du $4-9^{\circ}$ jour. Des constatations analogues sont faites à propos de C. burneti; du $8^{\mathrm{e}}$ au $18^{\mathrm{e}}$ jour après l'inoculation de particules filtrables, il y a vacuolisation du cytoplasme dans les cellules parasitées, agglomération de granulations, apparition de formes en mûres avant celle des formes typiques (Kordova et Rehaček, 1959, 201). La succession de ces diverses formes semble liée au développement et à la multiplication des rickettsies, et se fait toujours dans le même ordre.

La richesse en rickettsies augmente et n'est pas affectée par les métamorphoses. C'est au $210^{\circ}$ jour après l'infection qu'Amosenkova, Goldin et Daiter $(1961,664)$ trouvent la plus grande quantité de $C$. burneti dans les frottis de tube digestif de tiques par la méthode des anticorps fluorescents.

C'est par cette méthode qu'a été étudiée la distribution de $R$. rickettsi dans l'organisme de Dermacentor andersoni (Burgdorfer, 1961, 27). Chez les adultes frais éclos, les rickettsies sont bacilliformes ou lancéolées; au cours d'un jeûne de 4-6 mois, il n'y a plus que des formes sphériques, déterminant peu de fluorescence; mais après une incubation à $37^{\circ} \mathrm{C}$ ou 2 jours de repas sur cobaye, les formes bacillaires et lancéolées réapparaissent et sont les plus fréquentes (manifestant des fissions binaires); elles se trouvent dans presque tous les tissus (entre les fibres musculaires, dans les ganglions cérébroïdes, les cellules digestives, les glandes salivaires, les ovaires).

Il y a donc alternance de formes actives de multiplication (allongées) et de formes inactives quiescentes (sphériques) dans les conditions défavorables du jeûne.

La localisation des Wolbachia dans divers organes des Argasidae a été étudiée chez Argas arboreus et $A$. persicus (Roshdy, 1961, 148 ; Suitor, 1961, 95 et 1964, 111), et chez Ornithodorus moubata (Hecker, Aeschlimann et Burckhardt, 1968, 256) par les antigènes fluorescents ou par la microscopie électronique.

Lors de l'infection du pou par $R$. prowazeki ou des puces par $R$. mooseri, les rickettsies ne cultivent que dans les cellules de l'épithélium digestif; il n'y en a pas dans les restes de l'organisme ; elles sont éliminées par la voie fécale, où elles persistent longtemps; elles peuvent pénétrer facilement les muqueuses soit sous forme de souillure humide, soit se répandre en poussières et infecter les vertébrés par la voie aérienne.

Chez Ornithodorus moubata, $R$. prowazeki détermine une infection intestinale qui 
se conserve de nombreuses années au cours des mues et des repas (Weyer, 1954, 193 ; $1964,131)$. Avec $R$. conori chez le pou infecté par voie rectale, il y a culture de la rickettsie dans les cellules digestives, sans qu'il y ait hypertrophie ; lors d'inoculation parentérale, la rickettsie cultive sur place, puis envahit secondairement les cellules intestinales (Weyer, 1954, 205).

Chez les Argasidae, les rickettsies ne passent pas la barrière intestinale.

\section{Persistance des Rickettsies chez les Tiques.}

La survie et la multiplication des rickettsies se poursuivent durant toute la vie de l'hôte ; ceci traduit peut-être une relation très primitive entre les rickettsies et les arthropodes, alors que le parasitisme sur les vertébrés est secondaire. Le cas des Wolbachia maintient la situation primitive.

Chez les tiques infectées, la concentration en rickettsies est élevée ; les broyats de tiques en sont la source la plus riche. Ce sont également les réservoirs les plus commodes pour la conservation ou le transport des diverses espèces de rickettsies, qu'il $\mathrm{y}$ ait infection générale (Ixodida) ou intestinale (Argasida). Cette aptitude extrême oblige d'ailleurs à interpréter avec précaution les isolements de souches rickettsiennes à partir de tiques récoltées dans la nature, d'autant plus si les $x$ tiques sont gorgées; il est difficile dans ces conditions de savoir s'il s'agissait d'une infection réelle, avec implication possible de la tique dans un cycle épidémiologique, ou simplement une conservation ou un transport passif de sang infectieux chez la tique, et qui n'a pas d'autre signification qu'un isolement à partir de sang de vertébré, même si le procédé est plus commode. C'est ce qui se produit vraisemblablement avec les Rakeia sp. (Chlamydiaceae), éléments directement contagieux ordinairement, mais qui isolés fréquemment de broyats totaux de tiques, pourraient faire croire que ces dernières interviennent dans leur transmission.

La survie des rickettsies est possible même chez les cadavres de tiques, enrobées dans le contenu intestinal, au même titre que les poussières de matières fécales; des temps de conservation extrêmement longs ont été observés, jusqu'à 18-33 mois pour C. burneti (Weyer, 1953, 344), jusqu'à 14-33 mois pour $R$. rickettsi chez Ornithodorus parkeri (Davis, 1943, 1201).

\section{Pathologie des vecteurs par les Rickettsies.}

R. prowazeki parvient à tuer son vecteur, le pou.

Dans les conditions expérimentales, cette même rickettsie chez Dermacentor marginatus et $D$. reticulatus entraîne des morts prématurées de femelles, ou une faible production d'œufs, qui donnent peu de larves non viables ; or les rickettsies étaient en très grande quantité dans les organes de ces tiques (Piontkovskaja et Koršunova, 1953, 29).

Ordinairement, les puces survivent à leur infection par $R$. mooseri.

Suivant les souches chez le pou infecté par voie rectale, $R$. conori peut se révéler 
pathogène (souche de Marseille) ou bénigne (souches du Kenya, d'Afrique australe) (Weyer, 1952, 170).

\section{Infection transstadiale.}

C'est un phénomène normal chez les tiques ; il est facilement contrôlé au laboratoire par des repas successifs sur animaux sains. Ainsi l'infection de C. burneti a pu se transmettre 1300 jours au cours des repas et des mues chez Ornithodorus moubata (Weyer, 1953, 344). L'observation à partir des larves est plus malaisée, car elles sont plus difficiles à infecter, du fait de la faible quantité de sang infectieux ingéré. Chez Ornithodorus tholozani on a observé 70 p. 100 d'infections transstadiales à partir des larves avec $C$. burneti (Daiter et Amosenkova, 1961, 166). Pour $R$. rickettsi, il peut y avoir 90 p. 100 d'infection chez des nymphes à jeun issues de larves de Dermacentor andersoni gorgées de sang infectieux (Burgdorfer et Lackman, 1960, 241).

Après les repas, il y a accroissement de la concentration en rickettsies ; les pourcentages d'isolement à partir de stades à jeun sont moins élevés, dans les mêmes lots, quaaprès repas sur un hôte neuf. Cette réactivation des souches devient un procédé de laboratoire.

\section{Infection transovarienne des Rickettsies.}

Le phénomène est très fréquent entre tiques et rickettsies. Il est lié à l'infection générale des tiques, en particulier des ovaires et des ovocytes. Il est de règle chez $R$. burneti, $R$. conori, $R$. rickettsi chez les Ixodida; il a été observé chez Ehrlichia canis, chez $R$. prowazeki (Reiss-Gutfreund, 1957, 1-79, chez des Amblyomma). L'infection transovarienne de la descendance est obligatoire chez $R$. tsutsugamushi, puisque ses vecteurs Trombiculidae ne sont hématophages qu'au stade larvaire.

C'est sur plusieurs générations que ces infections transovariennes ont été observées, au cours de nombreuses années, chez $R$. conori (Haemaphysalis leachi, 3 générations; Géar, 1954, 158; Rhipicephalus sanguineus, sur plusieurs générations, 10 ans, Fiontkovskaja et Žmaeva, 1962, 196), chez R. rickettsi (Ornithodorus parkeri, 4 générations, Davis, 1943, 1201), chez C. burneti (Ornithodorus tholozani, nombreuses générations, Piontkovskaja et Żmaeva, 1962, 196).

Les changements de pourcentages d'infection transovarienne enre vecteurs différents correspond à des facteurs spécifiques; alors que Dermacentor andersoni présente un pourcentage très élevé, pour $R$. rickettsi, il n'atteint que $30-40$ p. 100 avec $D$. variabilis (Price, 1954, 292).

L'infection transovarienne des tiques est un facteur très important dans l'épidémiologie des rickettsioses, propre à ce groupe d'affections, très différentes en cela des viroses.

Curieusement, d'après la plus grande part des références, Cowdria ruminantium ne semble pas apte à l'infection transovarienne; sa possibilité de transmission par les Amblyomma est en accord avec ce fait. 


\section{Infection transcoïtale.}

Observée pour $R$. rickettsi chez Dermacentor andersoni (infection de la femelle par le mâle), ses conséquences sur l'épidémiologie semblent réduites, malgré leur intérêt théorique (Philips et Parker, 1933, 266).

\section{Transmission des Rickettsies.}

Les voies de contamination des vertébrés par les rickettsies sont très variées, même si les tiques peuvent jouer un rôle de réservoir. Sont transmises strictement lors de la piqûre $R$. tsutsugamushi (par les Trombiculidae), R. prowazeki (par souillure fécale du point de piqûre par le pou ; par piqûres de tiques). $C$. burneti se transmet par contact avec la tique, avec les poussières provenant de la dessiccation des matières fécales, mais également, par contact avec les sécrétions et excrétions des vertébrés malades. Chez $R$. rickettsi et $R$. conori l'infection se fait par piqûre, mais est également possible par souillure fécale de la piqûre, ou contact et écrasement de la tique.

Chez les Argasidae, la transmission par piqûre est inopérante, car les rickettsies ne passent pas la barrière intestinale; les cas d'infection positive où ils sont en cause doivent correspondre à des souillures. Chez Ornithodorus tholozani, l'infection transovarienne par $C$. burneti se produit à 70 p. 100 , mais seulement 20 p. 100 des piqûres sont infectantes (Daiter et Amosenkova, 1961, 166).

Bien que le fait de la transmission des rickettsies par piqûre soit connu depuis longtemps, on manque de données sur la transmission proprement dite par ce moyen. Dans la plupart des cas où des rickettsies qui passent par les voies transstadiale et transovarienne sont transmises aux vertébrés par piqûre, il faut tenir compte du rôle possible de contamination par les excréments, les poussières fécales ou le liquide coxal. Chez $C$. burneti, cet aspect de la contamination est certainement plus important que la piqûre elle-même.

\section{Changements de propriétés des Rickettsies chez les vecteurs.}

$R$. rickettsi présente 4 types de virulence; les souches $\mathrm{R}, \mathrm{S}$ et $\mathrm{T}$ peuvent donner une phase avirulente; les souches $U$ peuvent donner plusieurs phases peu virulentes; toutes ces phases correspondent à des changemens phénotypiques; après passage sur embryon de poulet, elles reviennent à la virulence d'origine, chacune selon sa virulence propre. Ainsi des souches hébergées par Dermacentor andersoni deviennent avirulentes après plusieurs mois au froid; leur concentration n'a pas diminué chez la tique ; leur suspension injectée à des cobayes donne des accès courts suivis d'immunité contre des souches virulentes; mais si les tiques sont mises 2 jours à $37^{\circ} \mathrm{C}$ ou passées sur embryon de poulet, elles reviennent à la virulence normale (Price, 1953, 118).

L'influence des mues sur la virulence des rickettsies ést liée à l'existencè de formés de quiescence ; une souche virulente ingérée par des nymphes peut être réisolée jusqu'à la métamorphose; les jeunes adultes ne donnent plus que des souches avirulentes $(R$. rickettsi, D. andersoni). 
Il en est de même après passage par la voie transovarienne ; les larves à jeun hébergent une souche peu ou non virulente, mais celle-ci se réactive après repas sur un animal neuf et le pourcentage des isolements s'élève.

Des différences de phases existent aussi chez $C$. burneti. La phase avirulente recouvre sa virulence en une génération chez Dermacentor marginatus; il en est de même avec $D$. reticulatus, inoculé par voie parentérale. Inversement, une souche virulente inoculée à $D$. reticulatus et à 1 . ricinus demeure inchangée au cours de ce passage ; la présence d'anticorps spécifiques chez les mammifères, et leur absence chez les tiques, doit jouer un rôle dans la perte de virulence et leur recouvrement (Brézina et Réhaček, 1963, 260). Par ailleurs, les passages de souches avirulentes de C. burneti chez Rhipicephalus sanguineus et Haemaphysalis concinna n'a pas redonné de virulence (Polozov et Pautov, 1961, 210).

\section{Infections mixtes à Rickettsies chez les Tiques; Interférences.}

Chez le cobaye, les souches faibles de $R$. rickettsi injectées à un titre 10-30 fois plus élevé qu'une souche virulente inoculée simultanément, protège contre cette dernière. Les résultats sont analogues si l'infection est réalisée par les tiques; des nymphes neuves de $D$. andersoni mises à piquer en même temps que 100 nymphes infectées d'une souche faible et 2 nymphes infectées de souche virulente, donnent des adultes qui transmettent uniquement la souche faible, qui passe également à la descendance (Price, 1953, 180).

Ce phénomène peut être une conséquence de l'interférence chez le cobaye. De toute façon, ces résultats font comprendre la restriction au développement de nouvelles souches virulentes dans la nature; les mutants n'ont que peu de chances de se développer en foyer (Rehaček, 1965, 1-24).

\section{Bibliographie}

Amosenkova (N. I.), Goldin (R. B.) et Daiter (A. B.), 1961. - Studies on experimental rickettsial infections with the aid of fluorescent antibodies. HII. Examination of ticks for Rickettsia burneti. Vopr. Virol., 7, 664-669 (in Russian).

BENDA (R.), 1958. - Experimentálni přenos čs. klíštové encefalitidy na kozy saním nakaženy'ch samiěek obecného klístete Ixodes ricinus L. Cesk. Epidemiol. Mikrobiol. Immunol., 7, 1-5.

-, - - - The common tick Ixodes ricinus L. as a reservoir and vector of tick-borne encephalitis. I. Survival of the virus (strain B3) during the development of the tick under laboratory conditions. J. Hyg. Epidemiol. Microbiol. Immunol., Prague, 2, 314-330.

BURGDORFER (W.), 1961. - Evaluation of the fluorescent antibody technique for the detection of Rocky Mountain spotted fever rickettsiae in various tissues. Pathol. Microbiol. Suppl., 24, 27-29. 
- et Lackman (D.), 1960. - Identification of Rickettsia rickettsii in the wood tick Dermacentor andersoni by means of fluorescent antibody. J. infect. Diseases, 107, 241-244.

Daiter (A. B.) et Amosenkova (N. I.), 1961. - The role of Ixodidae ticks on Q. fever. II. Infection of Ornithodoros papillipes Bir. with Coxiella burneti in the experimental conditions. Trud. Inst. Epidemiol. Mikrobiol. Pasteur, Leningrad, 23, 166-180 (in Russian).

Davis (G. E.), 1943. - Experimental transmission of the spotted fevers of the United States, Colombia and Brazil by the argasid tick Ornithodoros parkeri. Publ. Health Repts (U. S.), 58, 1201-1208.

Hecker (H.), Aeschlimann (A.) et Burckhardt (M. J.), 1968. - Contribution à la connaissance des symbiotes chez Ornithodorus moubata (Ixodoidea). Etude au microscope électronique. Acta tropica, 25, 256-262.

Hoogstrana (H.), 1966. - Ticks in relation to human diseases caused by viruses. Ann. Rev. Entom., 11, 261-308.

-, 1967. - Ticks in relation to human diseases caused by Rickettsia species. Ann. Rev. Entom., 12, 377-420.

Hurlbut (H. S.), 1956. - West Nile virus infection in arthropods. Amer. J. trop. Méd. Hyg., $5,76-85$.

Kordova (N.) et ReHacek (J.), 1964. - Microscopical investigations of organs from ticks infected with Rickettsia prowazeki. Acta. virol., 8, 465-469.

LEwIS (E. A.), 1946. - Nairobi sheep disease : the survival of the virus in the tick Rhipicephalus appendiculatus. Parasitology, 37, 55-59.

Libíkovâ (H.), RehÂčex (J.), Mayer (V.), KožUch (O.) et Ernek (E.), 1964. - Viruses of tick-borne encephalitis isolated from ticks lxodes persulcatus by means of different methods. J. Hyg. Epidemiol. Microbiol. Immunol., Prague, 8, 77-86.

MAC LeOD (J.), 1962. - Observations on tick transmission of louping-ill to sheep. J. comp. Path. Thérap., 72, 411-419.

Petriščeva (P. A.) et Levkovič (E. N.), 1949. - On the spontaneous virus carrier state of the Ixodes persulcatus and Ixodes ricinus ticks in new focus of tick-borne encephalitis. Vopr. krai. Obscestvo eksp. Parazitol., 4, 42-45 (in Russian).

PHILIP (C. B.), 1959. - Some epidemiological considerations in Rocky Mountain spotted fever. Publ. Health Repts (U. S.), 74, 595-600.

- et PARKer (R. R.), 1933. - Rocky Mountain spotted fever: investigation of sexual transmission in the wood tick Dermacentor andersoni. Publ. Health Repts (U.S.), 48, 266-272.

ProntKovskaja (S. P.) et Koršunova (O. S.), 1953. - Experiments of infection of Dermacentor marginatus Sulz. and Dermacentor pictus Herm. ticks with Rickettsia prowazeki. Vopr. krai. Obscestvo eksp. Parazitol., 8, 29-33 (in Russian).

— et Žmaeva (Z. M.), 1962. - Ixodid ticks. Vectors of agents in natural nidi. Med. gen. Izd., 342 pp. (in Russian).

Polozov (A. I.) et Pautov (V. N.), 1961. - The problem of the variation of Rickettsia burneti. Vopr. Virol., 6, 210-212 (in Russian). 
PrICE (W. H.), 1953. - A quantitative analysis of the factors involved in the variations of virulence of rickettsiae. Science, 118, 49-52.

- , - - - Interference phenomenon in animal infections with rickettsiae of Rocky Mountain spotted fever. Proc. Soc. exp. Biol. Med., 82, 180-184.

—, 1954. - The epidemiology of Rocky Mountain spotted fever. II. Studies on the biological survival mechanism of Rickettsia rickettsi. Amer. J. Hyg., 60, 292-319.

REHÂčEK (J.), 1959. - Přežívání virusu severo-americké encefalomyelitidy koní vychodnìho ty.pu (EEE) v klístatech Lxodes ricinus L. a Dermacentor marginatus Sulz. Biológia, $14,688-693$.

- 1960. - Experimental hibernation of the tick-borne encephalitis virus in engorged larvae of the tick Ixodes ricinus L. Acta virol., 4, 106-109.

-, 1962. - Transovarial transmission of the tick-borne encephalitis virus by ticks. Acta virol., 6, 220-226.

-, 1965. - Development of animal viruses and rickettsiae in ticks and mites. Ann. Rev. Entom., 10, 1-24.

REISS-GUtFREUND (R.-J.), 1957. - Un nouveau réservoir de virus pour Rickettsia prowazeki, les animaux domestiques et leurs tiques, Paris (Thèse pour le Doctorat ès-Sciences, A 734), $79 \mathrm{pp}$.

Roshdy (M. A.), 1961. - Observations by electron microscopy and other methods on the intracellular rickettsia-like microorganisms in Argas persicus Oken, 1818. J. Insect. Pathol., 3, 148-166.

Rozeboom (L. E.) et BURgdorfer (W.), 1959. - Development of Colorado tick fever virus in the Rocky Mountain wood tick Dermacentor andersoni. Amer. J. Hyg., 69, 138145 .

SUITOR (E. C.) et WEISS (E.), 1961. - Isolation of rickettsia-like microorganisms (Wolbachia persica n. sp.) from Argas persicus (Oken). J. infect. Dis., 108, 95-106.

-, 1964. - The relationship of Wolbachia persica Suitor et Weiss to its host. J. Insect. Pathol., 6, 111-124.

TARASEVIč (I. V.), 1957. - Study of experimental Q fever in Hyalomma plumbeum plumbeum Panz. Z. Mikrobiol. Epidemiol. Immunobiol., 28, 818-823 (in Russian).

VARMA (M. G. R.) et SMith (C. E. G.), 1960. - Studies on Langat virus (TP 21) in Haemaphysalis spinigera Neumann. Biology of viruses of the tick-borne encephalitis complex (Czechoslovak Acad. Sci. Press), 397-400.

Vermeil (C.), Lavillaureix (J.) et Reeb (E.), 1960. - Sur la conservation et la transmission du virus West Nile par quelques arthropodes. Bull. Soc. Pathol. exot., 53, 273-279.

WeyER (F.), 1952. - Die künstliche Infektion von Kleiderläusen und Mehlkäferlarven mit Rickettsia conori (Zeckenbissfieber). Acta trop., 9, 170-183.

-, 1953. - Die Beziehungen des Q-Fieber-Erregers (Rickettsia burneti) zu Arthropoden. Zeitschr. Tropenmed. Parasitol., 4, 344-382.

-, 1954. - Vergleichende Untersuchungen über das Verhalten verschiedener RickettsienArten in der Kleiderlaus. Acta trop., 11, 193-221. 
- , - - - Beobachtungen an einem Stamm von südafrikanischem Zeckenbissfieber. Zeitschr. Tropenmed. Parasitol., 5, 205-218.

-, 1959. - Experimente mit einem Stamm von Zeckenbissfieber aus Nordqueensland. Schweiz. Zeitschr. allgem. Pathol. Bakteriol, 22, 609-620.

-, 1964. - Experimentelle Uebertragung von Rickettsien auf Arthropoden. Zeitschr. Tropenmed. Parasitol., 15, 131-138.

Whitman (L.) et AitKen (T. H. G.), 1960. - Potentiality of Ornithodoros moubata Murray as a reservoir vector of West Nile virus. Ann. trop. Méd. Parasitol., 54, 192-204. 